\title{
Discrimination "toxic" to minorities, says public health expert
}

$\mathrm{S}$ ubtle, day-to-day racism may be a stronger driver of health inequalities than previously suspected, warns a professor at the Harvard School of Public Health in Boston, Massachusetts.

"The evidence to date suggests it's a toxic and largely neglected stressor that has pervasive, adverse effects over and above education and income," David Williams told members of the North American Primary Care Research Group at a Nov. 9 meeting in Ottawa, Ontario.

Between 2003 and 2006, racial health disparities cost the United States some $\$ 1.24$ trillion - a toll conventionally chalked up to differences in socioeconomic status, said Williams. In theory, that means a wealthy, educated black person should experience similar health outcomes to an equally wealthy and educated white person.

"Today, we know the pattern is much more complicated," said Williams. Even after controlling for factors such as income, education and occupation, "minorities have higher rates of diseases, get sick at younger ages, have more severe illness and die sooner than whites."

Williams cited towering infant mortality rates among African American women as a prime example. "The bestoff black women, those with a college degree, have the lowest infant mortality rate among African American women. But their rate is about three times as high as for other women with a college degree, and higher than the infant mortality rates for the worst-off white, Asian and Latina women who have not finished high school."

Although social status can have a powerful effect on health outcomes across racial groups, it appears moving up the ranks of society confers a lesser boon on minorities than their white peers. For example, "blacks and Hispanics receive less income at the same level of education, and have less wealth and purchasing power at equivalent income levels."

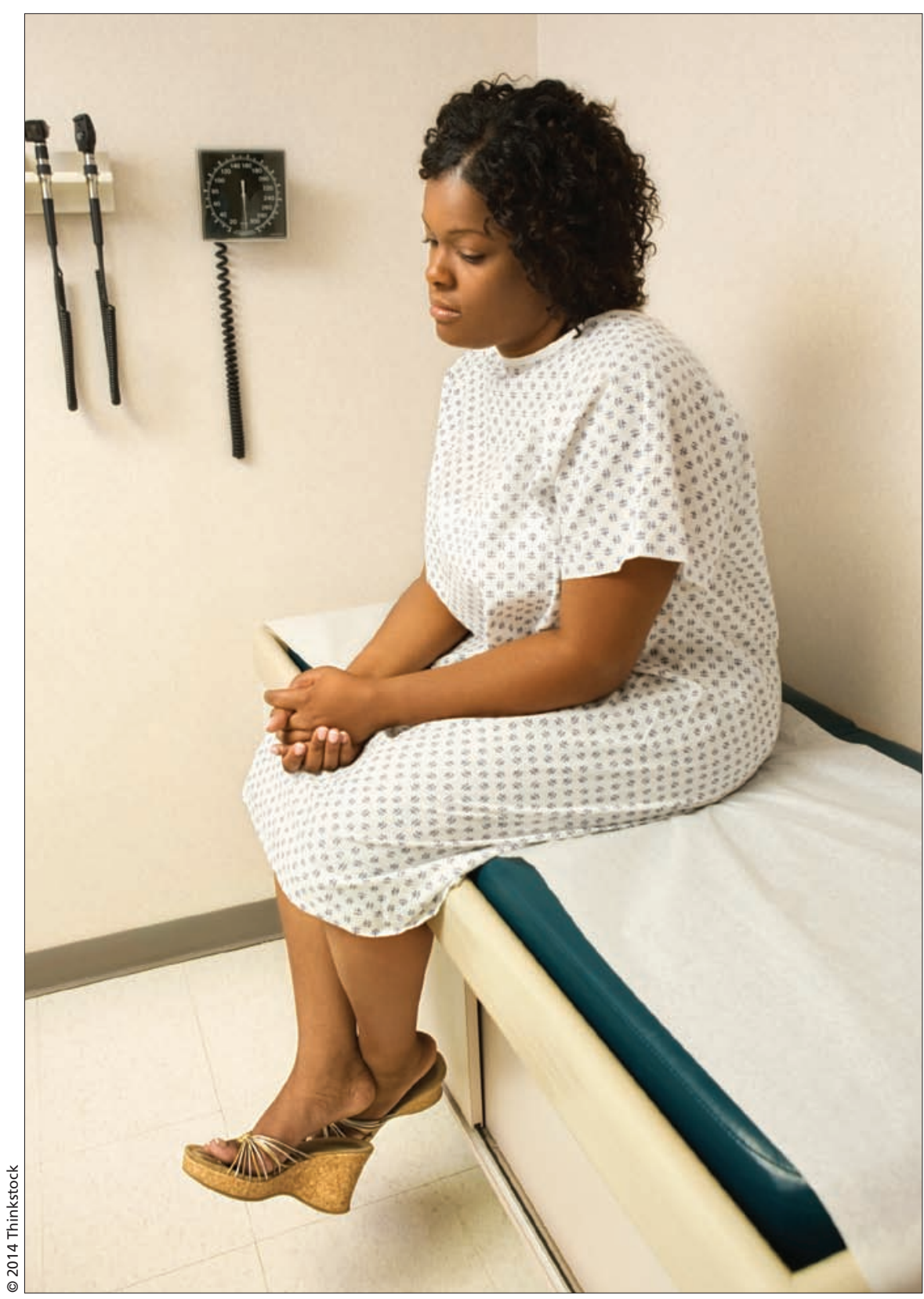

Mounting evidence suggests minority patients receive poorer quality medical care than whites, even when socioeconomic status is taken into account.

The same is true in other "race conscious" societies, including Canada, Australia, New Zealand and the United Kingdom, added Williams. "This profoundly says that there's something else about race, over and above economic status, that's driving variations in health."
Meanwhile, there's mounting evidence that personal experiences of "everyday discrimination" — such as receiving inferior service or being treated as less intelligent than others - can have diverse negative health impacts.

"People who report high levels of everyday discrimination are more likely 
to have coronary artery calcification, high levels of inflammation and high blood pressure," said Williams. "Women who report this discrimination during pregnancy give birth to lower-weight infants, and among the elderly, those who report high levels of discrimination experience more rapid decline, poorer sleep and increased mortality over time."

The prevalence of such discrimination in the health care system is particularly concerning, he said. "Across virtually every therapeutic intervention, ranging from the most high-tech procedures to the most elementary interventions, minority patients receive fewer procedures and poorer quality medical care than whites," and the pattern persists "even after differences in health insurance, socioeconomic status, stage and severity of disease, comorbidity and type of medical facility are taken into account."

Williams attributes the trend to providers' unconscious biases. 'I've talked to many medical groups about this, and the typical reaction is, 'I know I don't stereotype because I'm not racist.' But these processes are activated more quickly than cognition, so the people who say they would never do this are the people perfectly set up to do it."

The only way to counter such biases is to "consciously decide and take time to focus on the unique qualities of patients," he said. "These processes are not just about race; if you own negative stereotypes about gay, fat or old people, you need to be aware that you will unthinkingly treat them differently."

Williams also called for increased partnerships between primary care facilities and other community services to deliver "care within a social context."

He highlighted a successful medical-legal partnership at the Boston Medical Center as a cost-effective model. Primary care providers at the facility can refer patients to an on-staff lawyer, who in turn manages a volunteer legal team. "A child with asthma in a moldy apartment will not breathe symptom-free, regardless of the medication you give her, if the underlying living conditions are not addressed. A call from a lawyer can make a huge difference in terms of landlord response."

According to Williams, a three-year study of a similar program in Cincinnati, Ohio, showed that $89 \%$ of referrals led to positive outcomes, such as improved housing, benefits and education, helping both targeted patients and a much larger group of family members.

"Health system reform is crucial but insufficient to improve health," he urged. "We need to work actively with other sectors to bring resources together to modify where and how we live, learn, work and play, and in this process, attend first to those farthest behind." Lauren Vogel, CMAJ

CMAJ 2014. DOI:10.1503/cmaj.109-4661 